\title{
THE OVERALL ASSURANCE INTERVAL FOR THE NON-ARCHIMEDEAN EPSILON IN DEA MODELS
}

\author{
B. Daneshian ${ }^{1}$, G. R. Jahanshahloo ${ }^{2}$, F. Hosseimzadeh Lotfi ${ }^{3}$, T. Allahviranloo ${ }^{3}$ and S. \\ Mehrabian $^{2}$ \\ ${ }^{1}$ Dept. of Mathematics, Lahijan Branch, Islamic Azad University, Tehran, Iran. \\ ${ }^{2}$ Dept. of Mathematics, Teacher Training University, Tehran, Iran. \\ ${ }^{3}$ Dept. of Mathematics, Science \& Research Branch, Islamic Azad Univ, Tehran, Iran. \\ E-Mail: alahviranlo@yahoo.com, tofigh@allahviranloo.com
}

\begin{abstract}
Mehrabian, et. al. (1998) presented a procedure for determining overall assurance interval of $\varepsilon$. Solving $n$ linear programs are needed for this propose, where $n$ is the number of Decision Making Units involved in the evaluation. This paper proposes an efficient algorithm that can determine the overall assurance interval of $\varepsilon$ by solving a few number of linear programs.
\end{abstract}

Keywords- Data Envelopment Analysis, Non-Archimedean Infinitesimal.

\section{INTRODUCTION}

Charnes, Cooper and Rhodes (1978) published a breakthrough paper which pioneered the Data Envelopment Analysis (DEA) approach, a methodology for determining the relative efficiencies of Decision Making Units (DMU's). The data are input-output observations for a number of DMU's using varying amounts of the same inputs to produce varying amounts of the same outputs.

In recent years DEA has enjoyed both rapid growth and widespread acceptance. A bibliography by Emrouznejad and Thanassolis (1997) contains almost 1500 studies employing the methodology of DEA. In these studies the two most frequently used models are the Charnes, Cooper and Rhodes (CCR) (1978) model and the Banker, Charnes and Cooper (BCC) (1984), model, which both involve the NON-Archimedean infinitesimal, $\varepsilon$.

As a theoretical construct, $\varepsilon$ provides a lower bound for multipliers, to keep them away from zero. Some difficulties arise in representing an infinitesimal, because of finit tolerances in computer calculations.

Ali and Seiford (1993) have proposed an upper bound on $\varepsilon$ for feasibility of the multiplier side and boundedness of the envelopment side of the CCR and BCC models (see also Chapter 4 of Charnes, et. al. (1994)). Mehrabian, et. al. (1998) showed that Ali and Seiford's bound for $\varepsilon$ cannot be valid. They also provided a procedure for determining an assurance interval of the non-Archimedean $\varepsilon$. Solving $n$ linear programs are needed to determine the assurance interval where $n$ is the number of decision making units under evaluation. This paper presents an efficient algorithm that can determine the assurance interval of the non-Archimedean $\varepsilon$ by solving a few number of linear programs.

The paper is organized as follows. Section 2 presents definitions that are neede through the paper. This section also contains the procedure of determining the assurance interval of $\varepsilon$ presented by Mehrabian, et. al. (1998). Section 3 presents the new efficient algorithm of determining the assurance interval of $\varepsilon$. Section 4 continues with an empirical example. Concluding remarks appear in section 5. 


\section{DEFINITIONS}

Consider $n$ Decision Making Units (DMU`s), each consuming varying amounts of $m$ inputs in the production of s outputs. The $m \times n$ matrix of inputs is denoted by $X$ and the $s \times n$ matrix of outputs by $Y$. Further, $x_{i j}$ denotes the amount consumed of the $i$ th input by the $j$ th decision making unit and $y_{r j}$ denotes the amount produced of its $r$ th output. Finally, $X_{j}$ and $Y_{j}$ denotes, respectively, the vectors of inputs and outputs for the $j$ th DMU.

The input-oriented linear programming problem formulation for the $\mathrm{CCR}$ and $\mathrm{BCC}$ models for evaluation of $\mathrm{DMU}_{o}$ (both the envelopment and the multiplier side) are as follows:

$$
\begin{array}{lc}
C C R_{p}: \text { Envelopment Side } & C C R_{d}: \text { Multiplier Side } \\
\min \theta-\varepsilon\left(1 s^{o}+1 s^{i}\right) & \max U Y_{o} \\
\text { s.t }: & \text { s.t }: \\
\theta X_{o}-X \lambda-s^{i}=0, & V X_{o}=1 . \\
Y \lambda-s^{o}=Y_{o}, & U Y_{j}-V X_{j} \leq 0, \quad j=1, \ldots, n \\
\lambda \geq 0, s^{i} \geq 0, s^{o} \geq 0 & U \geq \varepsilon 1, V \geq \varepsilon 1 \\
\text { BCC } C_{p}: \text { Envelopment Side } & \text { BCC } C_{d}: \text { Multiplier Side } \\
\min \theta-\varepsilon\left(1 s^{o}+1 s^{i}\right) & \max U Y_{o}+u_{0} \\
\text { s.t } & \text { s.t. } \\
\theta X_{o}-X \lambda-s^{i}=0, & V X_{o}=1 . \\
Y \lambda-s^{o}=Y_{O} & U Y_{j}-V X_{j}+1 u_{0} \leq 0, \quad j=1, \ldots, n \\
1 \lambda=1 & U \geq \varepsilon 1, \quad V \geq \varepsilon 1 \\
\lambda \geq 0, \quad s^{i} \geq 0, s^{o} \geq 0 &
\end{array}
$$

Where $\varepsilon$ is an non-Archimedean infinitesimal.

Mehrabian, et. al. (1998) defined $P_{o}$ and $\bar{P}_{o}$ as the following LP problems corresponding to $\mathrm{DMU}_{o}$ :

$$
\begin{array}{lc}
P_{o}: \max \varepsilon & \bar{P}_{o}: \max \varepsilon \\
& \text { s.t. } \\
V X_{o}=1, & \text { s.t. } \\
U Y_{j}-V X_{j} \leq 0, \quad j=1, \ldots, n & U Y_{j}-V X_{j} \leq 0, \quad j=1, \ldots, n \\
U \geq \varepsilon 1, \quad V \geq \varepsilon 1 & U \geq \varepsilon 1, \quad V \geq \varepsilon 1
\end{array}
$$

where here $\varepsilon$ is a scalar variable. Note that the optimal solution of $P_{o}$ is equal to the optimal solution of $\bar{P}_{o}$. 
We review some definitions from Mehrabian, et. al. (1998).

Definition 1. The assurance interval for feasibility / boundedness of the CCR-model for the evaluation of $\mathrm{DMU}_{o}$ is defined as the interval $\left[0, \varepsilon_{o}^{*}\right]$, where $\varepsilon_{o}^{*}$ is the optimal value for $P_{o}$.

Definition 2. The intersection of all assurance interval for feasibility / boundedness of the CCR-model for the evaluation of all DMU's defines the overall assurance interval $\left[0, \varepsilon^{*}\right]$ with $\varepsilon^{*}=\min \left\{\varepsilon_{1}^{*}, \ldots, \varepsilon_{n}^{*}\right\}$.

Definition 3. Each element of the overall assurance interval $\left[0, \varepsilon^{*}\right]$ defines an assurance value of the non-Archimedean $\varepsilon$ for feasibility / boundedness of the CCRmodel for evaluation of all DMU's.

Based on Definition 2 the overall assurance interval $\left[0, \varepsilon^{*}\right]$ can be achieved by solving $n$ linear programs: $P_{1}, P_{2}, \ldots, P_{n}$.

Similar statements can be given for the BCC-model.

\section{A NEW ALGORITHM}

This section presents an algorithm for obtaining the overall assurance interval of the non-Archimedean $\varepsilon$ in the DEA models using a few linear programs, which is of computational important.

Consider the following LP problem:

$$
\begin{aligned}
& P^{\prime}: \max \varepsilon \\
& \text { s.t. } \\
& \quad V X_{j} \leq 1, \quad j=1,2, \ldots, n \\
& \quad U Y_{j}-V X_{j} \leq 0, \quad j=1,2, \ldots, n \\
& \quad U \geq \varepsilon 1, \quad V \geq \varepsilon 1 .
\end{aligned}
$$

Let $\left(\varepsilon^{o}, U^{o}, V^{o}\right)$ be the optimal solution of $P^{\prime}$. Mehrabian et. al. (1998) showed that $\varepsilon^{o}$ is a nonzero assurance value and also they showed that if $\left\{j: V^{o} X_{j}=1\right\}$ is singleton, then $\varepsilon^{o}=\varepsilon^{*}$, i.e., solving only one LP problem leads to the overall assurance interval of the non-Archimedean $\varepsilon$. Here, this idea will be extended.

Let $J_{p} \subseteq\{1, \ldots, n\}$, consider $P_{J_{p}}^{\prime}$ as the following LP problem:

$$
\begin{aligned}
P_{J_{p}}^{\prime}: & \max \varepsilon \\
& \text { s.t. } \\
& V X_{j} \leq 1, \quad j \in J_{p} \\
& U Y_{j}-V X_{j} \leq 0, \quad j=1, \ldots, n \\
& U \geq \varepsilon 1, \quad V \geq \varepsilon 1
\end{aligned}
$$

Lemma. If $\left(\varepsilon^{J_{p}}, U^{J_{p}}, V^{J_{p}}\right)$ is the optimal solution of $P_{J_{p}}^{\prime}$ and $\left\{j: V^{J_{p}} X_{j}=1\right\}=\{t\}$ is the singleton, then $\varepsilon^{J_{p}}=\min \left\{\varepsilon_{j}^{*}: j \in J_{p}\right\}$, where $\varepsilon_{j}^{*}$ is the optimal solution of $P_{j}$. 
Proof: Consider the dual of $P_{J_{p}}^{\prime}$ as follows:

$$
\begin{aligned}
& D_{J_{p}}^{\prime}: \min \sum_{j \in J_{p}} \theta_{j} \\
& \text { s.t. } \\
& \quad 1 W+1 Z=1 \\
& \quad \sum_{j \in J_{p}} \theta_{j} X_{j}-X \lambda-Z=0 \\
& \quad Y \lambda-W=0 \\
& \quad W \geq 0, Z \geq 0, \lambda \geq 0, \theta_{j} \geq 0, j \in J_{p} .
\end{aligned}
$$

By hypothesis, $V^{J_{p}} X_{j}<1, \quad j \in J_{p} \backslash\{t\}$. Then the complementary slackness condition implies that in the optimal solution of $D_{J_{p}}^{\prime}, \theta_{j}^{J_{p}}=0, \quad j \in J_{p} \backslash\{t\}$. Hence, $\theta_{t}^{J_{p}}$ is the optimal value of the following problem:

$$
\begin{array}{ll}
\min & \theta_{t} \\
\text { s.t. } & \\
& 1 W+1 Z=1 \\
& \theta_{t} X_{t}-X \lambda-Z=0 \\
& Y \lambda-W=0 \\
& W \geq 0, Z \geq 0, \lambda \geq 0, \theta_{t} \geq 0
\end{array}
$$

and this is a dual problem for $\bar{P}_{t}$, which has the optimal value of $\varepsilon_{t}^{*}$, then $\varepsilon^{J_{p}}=\varepsilon_{t}^{*}$. Since $\varepsilon^{J_{p}} \leq \min \left\{\varepsilon_{j}^{*}: j \in J_{p}\right\}$, then $\varepsilon^{J_{p}}=\min \left\{\varepsilon_{j}^{*}: j \in J_{p}\right\}$.

Extension of the foregoing lemma is trivially leaded to the following theorem:

Theorem. Let $J_{1}, J_{2}, \ldots, J_{k}$ be $k$ subset of $\{1,2, \ldots, n\}$ such that $J_{1} \cup J_{2} \cup \ldots \cup J_{k}=\{1,2, \ldots, n\}$ and $\left(\varepsilon^{J_{p}}, U^{J_{p}}, V^{J_{p}}\right)$ be the optimal solution of $P_{J_{p}}^{\prime}$ for which $\left\{j: V^{J_{p}} X_{j}=1\right\}$ is singleton, for all $p, p=1,2, \ldots, k$, then

$$
\varepsilon^{*}=\min \left\{\varepsilon^{J_{1}}, \varepsilon^{J_{2}}, \ldots, \varepsilon^{J_{k}}\right\}
$$

As a result of the theorem is to construct the following efficient algorithm that is used for finding the overall assurance interval of the non-Archimedean $\varepsilon$ :

Step 0: Set $T=\{\}, \quad J=\{1,2, \ldots, n\}$.

Step 1: Solve $P_{J}^{\prime}$ and find its its optimal solution: $\left(\varepsilon^{J}, U^{J}, V^{J}\right)$.

Step 2: Find $K=\left\{j: V^{J} X_{j}=1\right\}$

If $|K|=1$

then $T \leftarrow T \cup K$ and continue from Step 3,

else $J \leftarrow J \backslash K, \quad T \leftarrow T \bigcup K$ and come back to Step 1 .

Step 3: Solve $P_{j}$ and save its optimal solution $\varepsilon_{j}^{*}$, for all $j, j \in T$.

Step 4: $\varepsilon^{*}=\min \left\{\varepsilon_{j}^{*}: j \in T\right\}$. 


\section{EMPIRICAL EXAMPLE}

The algorithm of determining the overall assurance interval of $\varepsilon$ is illustrated by means of the 44-unit data set of table 1 from Alirezai and Alamdar (1998). The data are concerned with 44 Power Plant Systems of Iran for the year 1997 and consist of three outputs (units performance factor, capability factor, and availability factor) and two inputs (production conditions and number of personnel).

The algorithm implication leads the following results:

$$
T=\{33,42,39,41,13,38,12,40,23,25,44\}
$$

The following table presents $\varepsilon_{j}^{*}$ for $j \in T$.

\begin{tabular}{cc}
\hline$j$ & $\varepsilon_{j}^{*}$ \\
\hline 33 & 0.0025465641 \\
42 & 0.0028170620 \\
39 & 0.0027096794 \\
41 & 0.0025388063 \\
13 & 0.0037240236 \\
38 & 0.0025148081 \\
12 & 0.0026216156 \\
40 & 0.0027549076 \\
23 & 0.0026506155 \\
25 & 0.0025719935 \\
44 & 0.0026071612 \\
\hline
\end{tabular}

Then

$$
\varepsilon^{*}=\min \left\{\varepsilon_{j}^{*}: j \in T\right\}=0.0025148081
$$

Hence, the overall assurance interval is $\left[0, \varepsilon^{*}\right]=\left[\begin{array}{ll}0,0.0025148081\end{array}\right]$.

Note that the foregoing algorithm gives the overall assurance interval of nonArchimedean $\varepsilon$ by solving a few number of LP problems and it is computationally efficient in comparison with procedure presented by Mehrabian, et. al. (1998) in which solving $n$ LP problems were needed.

\section{CONCLUSION}

This paper presented an efficient algorithm for determining the overall assurance interval of the non-Archimedean $\varepsilon$ in DEA models. Solving a few number of LP problems are needed for the propose of the algorithm while for the procedure presented by Mehrabian, et. al. (1998), $n$ LP problems have to solve- where $n$ is the number of units under evaluation.

An empirical test using 44 units data set confirmed the capability of proposed algorithm 
Table 1: The data for 44 Power Plants Systems.

\begin{tabular}{c|ccccc}
\hline DMU`s & $I_{1}$ & $I_{2}$ & $O_{1}$ & $O_{2}$ & $O_{3}$ \\
\hline 1 & 1.06 & 0.333 & 88.54 & 64.35 & 86.27 \\
2 & 1.05 & 0.72 & 89.76 & 71.1 & 89.72 \\
3 & 1.07 & 0.4 & 89.96 & 55.62 & 85.68 \\
4 & 1.02 & 0.7 & 89.13 & 82.95 & 90.43 \\
5 & 1.07 & 0.498 & 78.32 & 75.99 & 75.57 \\
6 & 1.14 & 0.55 & 73.36 & 73.81 & 61.01 \\
7 & 1.05 & 0.75 & 90.22 & 79.65 & 89.97 \\
8 & 1.21 & 0.516 & 79.87 & 76.38 & 65.97 \\
9 & 1.19 & 0.525 & 77.21 & 39.981 & 82.94 \\
10 & 1.23 & 0.363 & 73.34 & 45.048 & 88.88 \\
11 & 1.24 & 0.612 & 78.34 & 84.61 & 76.11 \\
12 & 1.48 & 1.563 & 74.54 & 37.5 & 69.03 \\
13 & 1.03 & 4.15 & 89.39 & 83.34 & 77.9 \\
14 & 1.15 & 3.32 & 83.36 & 66.38 & 81.33 \\
15 & 1.23 & 1.007 & 67.05 & 92.8 & 76.81 \\
16 & 1.19 & 0.959 & 80.4 & 81.21 & 87.87 \\
17 & 1.28 & 0.937 & 68.84 & 74.42 & 77.00 \\
18 & 1.29 & 0.694 & 70.38 & 73.13 & 57.07 \\
19 & 1.36 & 0.32 & 81.29 & 92.45 & 85.66 \\
20 & 1.33 & 0.314 & 88.92 & 74.99 & 89.98 \\
21 & 1.31 & 0.479 & 86.88 & 57.78 & 89.33 \\
22 & 1.41 & 0.313 & 73.42 & 73.28 & 69.37 \\
23 & 1.55 & 0.81 & 33.05 & 46.25 & 64.76 \\
24 & 1.32 & 0.635 & 61.1 & 49.68 & 82.4 \\
25 & 1.51 & 1.222 & 57.95 & 77.63 & 62.6 \\
26 & 1.49 & 0.347 & 34.09 & 96.94 & 71.35 \\
27 & 1.38 & 0.347 & 90.25 & 80.57 & 90.49 \\
28 & 1.31 & 0.78 & 50.66 & 66.28 & 83.54 \\
29 & 1.39 & 0.304 & 83 & 56.57 & 89.73 \\
30 & 1.31 & 0.625 & 65.63 & 88.93 & 59.12 \\
31 & 1.46 & 05 & 43.77 & 77.78 & 74.06 \\
32 & 1.49 & 0.6 & 73.92 & 70.93 & 77.35 \\
33 & 1.67 & 0.711 & 12.77 & 78.07 & 96.43 \\
34 & 1.34 & 0.732 & 82.74 & 66.38 & 88.03 \\
35 & 1.41 & 0.25 & 90.44 & 50.55 & 55.05 \\
36 & 1.42 & 0.346 & 92.35 & 57.16 & 62.23 \\
37 & 1.37 & 0.444 & 82.04 & 74.68 & 41.65 \\
38 & 1.61 & 0.909 & 94.49 & 70.72 & 51.39 \\
39 & 1.43 & 2 & 99.36 & 94.08 & 54.69 \\
40 & 1.6 & 0.526 & 87.6 & 65.14 & 26.88 \\
41 & 1.64 & 0.795 & 76.11 & 68.6 & 24.5 \\
42 & 1.37 & 3.333 & 100 & 57.39 & 48.82 \\
43 & 1.43 & 0.588 & 91.29 & 91.18 & 42.1 \\
44 & 1.49 & 1.111 & 89.43 & 100 & 47.12 \\
\hline & & & & &
\end{tabular}




\section{REFERENCES}

1. A. I. Ali, L. M. Seiford, "Computational accuracy and infinitesimals in Data Envelopment Analysis," INFOR, Vol. 31, No. 4, pp. 290-297, 1993.

2. M. R. Alirezaei, N. Alamdar, "Operation evaluation of Power Plants and Measuring their Technical Efficiencies with DEA," Paper Presented at the $13^{\text {th }}$ International Power System Conference, Tehran, Iran, 1998.

3. R. D. Banker, A. Charnes, W.W. Cooper, " Some models for Estimating Technical and Scale Inefficiencies in Data Envelopment Analysis," Management Science, Vol. 30, No. 9, pp. 1078-1092, 1984.

4. A. Charnes, W. W. Cooper, A. Y. Lewin, L. M. SEIFORD, data Envelopment Analysis: Theory, Methodology, and Applications, Boston: Kluwer Academic Publishers, 1994.

5. A. Charnes, W. W. Cooper, E. Rhodes, " Measuring the Efficiency of Decision Making Units," European Journal of Operations Research, Vol. 2, No. 6, pp. 429-444, 1978.

6. A. Charnes, J. Rousseau, Semple, “ An effective non-Archimedean anti-degeneracy / cycling linear programming method especially for data envelopment analysis and like methods," Annals of Operations Research, Vol. 47, pp. 271-278, 1993.

7. A. Emrouznejad, E. Thanassolis, "An extensive Bibliography of Data Envelopment Analysis Volume II," Journal Papers, University of Warwick, 1997.

8. S. Mehrabian, G. R. Jahanshahloo, M. R. Alirezaei, G. R. Amin, “ An Assurance Interval of the Non-Archimedean Epsilon in DEA Models," European Journal of Operations Research, Vol. 48, No. 2, pp. 344-347, 1998. 